\title{
Les parlers francoprovençaux de la Suisse romande : processus de patrimonialisation
}

Federica Diémoz

\section{(2) OpenEdition}

Édition électronique

URL : http://journals.openedition.org/esp/652

DOI : 10.4000/esp.652

ISSN : 2532-0319

Éditeur

Centre d'Information sur l'Éducation Bilingue et Plurilingue

\section{Édition imprimée}

Date de publication : 1 décembre 2015

Pagination : 71-78

ISSN : 1127-266X

\section{Référence électronique}

Federica Diémoz, «Les parlers francoprovençaux de la Suisse romande : processus de

patrimonialisation », Éducation et sociétés plurilingues [En ligne], 39 | 2015, mis en ligne le 01 octobre 2016, consulté le 20 avril 2019. URL : http://journals.openedition.org/esp/652 ; DOI : 10.4000/esp.652 


\section{LES PARLERS FRANCOPROVENÇAUX DE LA SUISSE ROMANDE: PROCESSUS DE PATRIMONIALISATION}

\section{Federica DiÉMOZ}

In una regione linguistica della Sizizera romanda, il cantone del Vallese, l'interruzione nella trasmissione delle lingue «tradizionalì (le parlate francoprovenzali) crea delle dinamiche di valorizzazione. Il sentimento di perdita trasforma una realtà linguistica e culturale un tempo denigrata in patrimonio da valorizzare. Si osservano diverse azioni di tutela del patrimonio culturale, sia da parte delle istituziom locali sia delle associazioni. Se queste attività stanno modificando gli atteggiamenti verso le lingue locali, la sfida sarà di vedere se anche le loro funzioni e le pratiche linguistiche reali saranno modificate all'interno di comunità bi-plurilingui.

\section{Parole chiavi: Sociolinguistica, Svizzera romanda, francoprovenzale, patrimonio linguistico}

In a part of French-speaking Swrtzerland, the Valais canton, the break in the transmission of so-called "traditional" languages (the Francoprovençal dialects), has launched a process to save them. The sense of loss has transformed a previously, looked down upon linguistic and cultural reality into a value. Thus several forms of heritage enhancement, from the "top" and from the "bottom", are surfacing. Though this is slowly changing people's attitudes towards the patois, the challenge will be to see how the identity and emotional niche which they presently occupy will be modified in today's bi-plurilingual community.

Key-words: Sociolinguistics, French-speaking Srertzerland, Francoprovençal, linguistic heritage

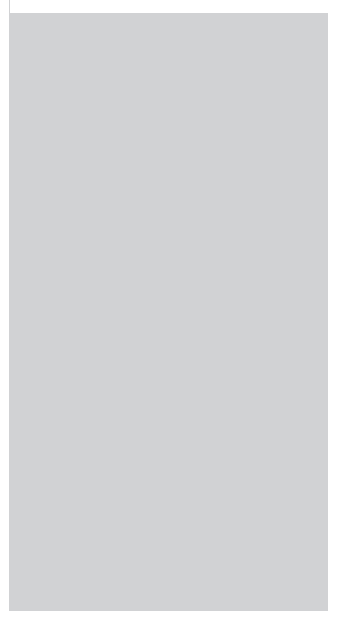

es langues autochtones forment une partie constituante du patrimoine linguistique de la Suisse romande, tout en n'étant pas reconnues officiellement au niveau fédéral. En effet, la constitution fédérale suisse prévoit que: «Les langues officielles de la Confédération sont l'allemand, le français et l'italien. Le romanche est aussi langue officielle pour les rapports que la Confédération entretient avec les personnes de langue romanche» (Art. 70.1 Langues). Les langues autochtones sont marginalement citées dans l'article suivant (Art. 70.2):

«Les cantons déterminent leurs langues officielles. Afin de préserver l'harmonie entre les communautés linguistiques, ils veillent à la répartition territoriale traditionnelle des langues et prennent en considération les minorités linguistiques autochtones».

Dans la constitution du canton du Valais de 1907, on ne mentionne pourtant que l'allemand et le français. Par contre, dans la constitution jurassienne de 1977, le dialecte franc-comtois fait son apparition comme 
Les parlers francoprovençaux de la Suisse romande: processus de patrimonialisation

F. DiÉMOz faisant partie du patrimoine jurassien. C'est dans le cadre des activités culturelles (Art. 42) qu'on lit (Article 42.2):

«lls ('État et les communes) veillent et contribuent à la conservation, à l'enrichissement et à la mise en valeur du patrimoine jurassien, notamment du patois».

Jusqu'à une époque relativement récente (fin XIXe-début XXe siècle), les parlers francoprovençaux et oiliques ont été le véhicule de la communication orale quotidienne en Suisse romande, dans toutes les régions, rurales et urbaines, et dans tous les milieux sociaux. Le français qui avait été introduit en Suisse romande à partir du XIIIe siècle sous forme écrite, commencera à faire son apparition à l'oral à partir du XVIIe siècle. Le recul des langues autochtones, appelées couramment patois par les locuteurs, s'explique par un ensemble de facteurs concomitants, politiques, économiques, sociaux et idéologiques. L'évolution linguistique et culturelle qui s’impose en France au lendemain de la Révolution de 1789 a accentué ce déclin. Le jacobinisme linguistique, le mépris et la politique de répression à l'égard de toutes les langues régionales et des dialectes a été imitée par les milieux intellectuels de la Suisse romande.

Ces langues dites traditionnelles, disparues au début du XXe siècle dans les cantons de Genève, Vaud et Neuchâtel, sont encore utilisées par la dernière génération de locuteurs jurassiens, fribourgeois et valaisans. Dans la plupart des villages, les dialectophones - tous bilingues - ont près de soixante-dix ans, voire plus. Dans ces situations particulières, on parle d'une situation de diglossie linguistique (Lüdi 1990) où la variété haute (le français) domine sur la variété basse (le dialecte), dont l'emploi est limité à certains contextes. Nous ne disposons pas de chiffre officiel sur le nombre effectif des locuteurs patoisants - actifs, passifs et néo-locuteurs (1) - et les données des recensements fédéraux ne peuvent pas être utilisées: soit la question de l'emploi des langues autochtones n'a pas été posée, soit elle a été mal interprétée. Les gens confondent facilement la variété régionale du français avec la langue locale historique, ce qui donne, par exemple, des chiffres complètement faux pour des locuteurs francoprovençaux dans le canton de Neuchâtel (Kristol 1998). Il s'agit toutefois d'un très petit nombre. La transmission «naturelle», quotidienne, dans la famille de générations en générations, a complètement disparu en Suisse romande, sauf dans quelques familles en Valais, en particulier dans la commune d'Évolène (Maître et al. 2004).

Plus récemment, ces langues locales ont trouvé un espace, même s'il est ambigu, de reconnaissance patrimoniale cantonale et fédérale à travers la mise en place de la convention sur la sauvegarde du patrimoine culturel immatériel de l'Unesco que la Suisse a ratifiée en 2008. Dans le cadre de l'Inventaire de l'Unesco sur le patrimoine culturel immatériel (PCI), les cantons suisses ont proposé 387 pratiques «traditionnelles» à la Confédération 
Les parlers francoprovençaux de la Suisse romande: processus de patrimonialisation

F. DiÉMOz helvétique. En Suisse, l'appellation de «patrimoine culturel immatériel» a été remplacée par "traditions vivantes», ce qui indique une certaine volonté de rester attaché à des traditions historiques du pays (Graezer Bideau 2012). Dans la liste définitive des traditions vivantes de Suisse retenue par l'Office Fédéral de la Culture en septembre 2011, les langues autochtones font leur apparition par le biais du théâtre en Valais «Patois $\mathrm{du}$ Valais romand en scène» et comme «Pratiques des patois jurassiens» dans le Jura. Dans le canton de Fribourg c'est «Le chant du Ranz des vaches» qui a été inscrit. Rappelons que dans la Convention pour la sauvegarde du PCI, les traditions et les expressions orales apparaissent comme un des domaines dans lesquels se manifeste le PCI et que les langues y figurent comme vecteur. C'est pour cette raison que les langues en tant que telles ne figurent pas dans l'inventaire, mais plutôt les pratiques ou les activités véhiculées par les langues (Diémoz et al. 2014).

Dans une région de la Suisse romande, le Valais, la rupture dans la transmission des langues dites «traditionnelles» au cours du XXe siècle, et en même temps des valeurs affectives et identitaires qu'elles véhiculent, a lancé un processus de sauvegarde à plusieurs facettes. De plus, le sentiment de perte transforme une réalité linguistique et culturelle, autrefois dénigrée, en un "patrimoine» à mettre en valeur (Ciarcia 2006, Rautenberg 2003).

«Il se dégage des discours de nos interlocuteurs une tendance nette quant au changement d'attitude intervenu ces dernières années face au patois. Autrefois minorisé et décrié, notamment par linstitution scolaire, lorsqu'il était la langue vernaculaire de toute la communauté, il se charge aujourd'hui de connotations positives auprès des locuteurs patoisants. [...] Cette évolution s'explique, selon un de nos interlocuteurs âgé d'une quarantaine d'années, par le fait que dans un monde qui se globalise, les gens ont davantage besoin de sentir leurs racines. Le patois contribue à assouvir ce besoin. Enfin, les attitudes changent aussi à l'extérieur de la commune d'Evolène. Une femme nous raconte ainsi qu'avant, lorsque les Evolénardes descendaient dans la capitale valaisanne avec leurs enfants, si elles parlaient patois, on se retournait en les regardant d'un air moqueur. Maintenant, les gens se retournent toujours mais ils sont positivement curieux, ils demandent "mais quelle langue parlez-vous?"》 (Maitre et al. 2004: 384).

Depuis les années 2000, on assiste dans le canton du Valais à une importante prise en compte politique et institutionnelle du patrimoine linguistique et culturel francoprovençal et une reconnaissance des erreurs commises autrefois par les institutions. Le Conseiller d'État Claude Roch, à l'occasion de la fête du cinquantenaire de la Fédération Cantonale Valaisanne des Amis du Patois, souligne que :

«Malgré les coups portés à la fin du XIXe siècle, et dans les 
Les parlers francoprovençaux de la Suisse romande: processus de patrimonialisation

F. DiÉMOz premières décennies du XXe, par une Instruction Publique soucieuse de satisfaire aux exigences de la modernité, le patois a survécu. Mieux, nous constatons aujourd'hui, en dépit de l'avance inexorable de la standardisation, un regain d'intérêt chez les jeunes pour la langue à la fois rude et franche, subtile et savoureuse de nos aïeux. [...] Au nom du Département de l'éducation, de la culture et du sport qui a parfois méconnu ce patrimoine, je suis heureux de m'associer à la fête du cinquantenaire de la Fédération et je lui adresse les voeux les plus chaleureux pour la continuation de sa méritoire activité» (Carthoblaz et al. 2004: 7).

En 2008 un Conseil du patois a été institué par le Conseil d'État, afin de développer un concept et un plan d'action pour valoriser le patrimoine que constitue le francoprovençal en Valais. L'année 2011 a marqué un tournant en faveur d'une certaine «reconnaissance officielle» des parlers francoprovençaux valaisans: la création de la Fondation pour le développement et la promotion du patois francoprovençal en Valais par l'État du Valais et la Fédération Cantonale Valaisanne des Amis du Patois qui fait suite et prolonge les activités du Conseil du patois.

De nombreux matériaux ont ainsi été réalisés (livres/BD, CD) et en particulier, la méthode pédagogique EOLE et patois. Éducation et ouverture aux langues patrimoniales (EOLE 2012) avec l'élaboration d'activités didactiques en lien avec différents patois francoprovençaux et oiliques et avec le français régional. Cet ouvrage a été édité par l'Institut de recherche et de documentation pédagogique (IRDP) de Neuchâtel et est le résultat d'un travail de collaboration entre une équipe de pédagogues de l'IRDP et une équipe de dialectologues du Centre de dialectologie et d'étude du français régional de l'université de Neuchâtel.

Le Conseil du patois souhaitait disposer d'un outil pédagogique à proposer aux écoles qui, jusqu'à présent, ne prenaient pas en compte les langues historiques. Toutefois, étant donné la situation sociolinguistique actuelle et la fonction plutôt de niche (Haugen 1972, Boudreau et alii. 2002) que ces langues occupent, c'est dans le cadre de la diversité linguistique et culturelle, de la comparaison entre langues romanes, que l'école peut prendre en compte les langues patrimoniales et contribuer à la construction de capacités d'apprentissage et de références culturelles chez les élèves. C'est donc dans cette optique que la méthode EOLE et patois a été conçue: non un enseignement de différentes variétés linguistiques de la Suisse romande et des autres régions galloromanes, mais une éducation et une ouverture à ces langues (Diémoz 2014). L'ensemble des documents EOLE et patois peut être consulté et téléchargé sur le site EOLE développé à l'IRDP:

http://www.irdp.ch/eole/eole_patois/index.html. En outre, certains 
Les parlers francoprovençaux de la Suisse romande: processus de patrimonialisation

F. DiÉMOz documents et matériaux ne figurent pas dans l'ouvrage mais sous forme de fichiers informatisés accessibles uniquement en ligne (les DocumentsÉlèves, les Documents et les Annexes liés à chaque activité). Dans les écoles suisses romandes, il n'est toutefois pas encore usuel de traiter la proximité des langues, de valoriser les ressources communes aux langues issues de la même famille linguistique, à savoir l'appartenance commune aux langues romanes pour la variété du français et les langues locales. On pourrait s'interroger également sur les représentations et les attitudes du corps enseignant à l'égard des parlers francoprovençaux qui connaissent actuellement une certaine reconnaissance et une valorisation importante: à ce propos, aucune enquête récente n'a été réalisée.

A côté de ce processus de patrimonialisation par le «haut» - les instances politiques, les élites scientifiques et culturelles - c'est aussi par le «bas», par un «mouvement de patrimonialisation ordinaire» (Hertz et al. 2012: 8-9) - associations de patoisants, associations culturelles bénévoles - que l'on observe une volonté croissante d'appropriation ou réappropriation d'une «identité» culturelle et linguistique qui traduit une volonté d'en empêcher ou d'en diminuer la perte. En particulier, les nombreuses associations valaisannes des amis du patois ont joué un rôle important au cours du XXe siècle, ainsi que les enseignements périodiques pour adultes dans certaines antennes de l'université populaire valaisanne, activités qui continuent de nos jours.

En nous basant sur des témoignages recueillis lors d'enquêtes dialectologiques et sociolinguistiques, menées dans le canton du Valais en 2011 et 2012 auprès de (néo-)patoisants membres d'associations plus ou moins récentes, nous allons essayer de décrire comment la perception des dialectes comme patrimoine s'inscrit dans cette histoire culturelle et politique des particularismes linguistiques en Suisse. Ces témoignages, étudiés dans un travail récent (Diémoz, Reusser-Elzingre 2014), permettent de résumer ici les principaux thèmes qui ont émergé.

Tout d'abord, le besoin de partager ce «patrimoine» autrefois commun à toute la communauté et de se rencontrer pour parler le patois pousse des locuteurs que nous avons interrogés à organiser des soirées de contage, de récits pour ne pas perdre leur langue de l'enfance et ainsi tenter de sauvegarder leur dialecte. Le lien nostalgique avec l'enfance est encore plus fort chez les locuteurs passifs, qui regrettent de n'avoir pas appris cette langue et sont demandeurs de cours de patois qu'ils souhaitent transmettre à la jeune génération.

Ensuite, le récit de la fin des patois, dans la bouche des locuteurs semble inexorable. Certains locuteurs essaient d'en détourner l'usage vers le privé et l'intime, la question et l'importance de leur survie sont indissociablement liées aux actions patrimoniales mises en place. Aux discours de la perte s'associent alors les éléments classiques de la mise en patrimoine: 
Les parlers francoprovençaux de la Suisse romande: processus de patrimonialisation

F. DiÉMOz le risque de rupture intergénérationnelle, la fin de la transmission culturelle, le renversement des stigmates du déni, la nécessité de la sauvegarde. Certains locuteurs passifs ou néo-locuteurs - plus jeunes et souvent des enseignants - sont très soucieux de la disparition de la langue, parfois plus que certains locuteurs natifs ou des spécialistes qui semblent mieux l'accepter.

Les témoignages attestent bien que les parlers francoprovençaux occupent la niche écologique de la sphère familiale, de la communication intime où les sentiments jouent un rôle essentiel. Ils remplissent également les fonctions de langue secrète ou de langue de cohésion qu'on utilise afin de ne pas être compris, pratique qui était bien répandue au sein des foyers - mais qui n'était pas si efficace (grâce aux compétences passives des jeunes) - et qui persiste encore de nos jours dans certains contextes, par exemple entre moniteurs de ski en Valais.

Les acteurs de la revitalisation participent d'un effort collectif qui tente de déplacer l'image négative des parlers vers une sauvegarde réaliste. L'enjeu n'est pas un retour triomphant du patois dans un activisme patrimonial, mais il s'agit d'entretenir la langue, de conserver la trace, de ne pas la laisser se perdre. Ils essaient d'en sauvegarder le maximum à travers la rédaction de dictionnaires ou la collecte d'archives sonores.

En ce qui concerne l'enseignement des parlers francoprovençaux, nous avons déjà pu mettre en relief dans d'autres contributions que les différents acteurs sociaux ont cependant des opinions très partagées. Certains politiciens et promoteurs y voient une obligation de l'institution scolaire - «'école doit refaire ce qu'elle a défait» - et cherchent à mettre en œuvre une pédagogie spécifique en tentant de retrouver l'«authenticité» de la langue; d'autres pensent que l'enseignement du patois n'est pas une solution, «enseigner le patois c'est le faire mourir plus vite». Selon les nouveaux acteurs partisans d'un apprentissage du patois, la transmission actuelle de la langue ne peut se faire qu'à l'école, à travers des cours de langue ou des rencontres de patoisants, puisque la transmission «naturelle», quotidienne dans la famille et intergénérationnelle, a presque complètement disparu. Au rôle de l'enseignement il faudrait ajouter celui que les médias pourraient jouer dans ce contexte de valorisation linguistique. Nous rappelons que depuis 2009 la radio et télévision valaisanne consacrent chaque semaine une rubrique au patois.

Dans le processus de patrimonialisation locale de la région francophone du Valais, on constate en général un intérêt croissant pour le parler des générations précédentes, qui se traduit par des représentations positives aux antipodes des jugements négatifs dont l'institution scolaire avait pétri l'imaginaire linguistique de leurs aînés. Par ailleurs, les locuteurs patoisants - qui ne doivent plus se taire - sont maintenant fiers et conscients de la richesse patrimoniale qu'ils veulent partager avec la communauté. 
Les parlers francoprovençaux de la Suisse romande: processus de patrimonialisation

F. DiÉMOz

BIBLIOGRAPHIE
De plus, les différents détenteurs prennent la parole à propos de la valorisation et de la sauvegarde du patois, mais aussi de leur identité communautaire. Ce renversement du stigmate est classique dans toutes les patrimonialisations ordinaires, qu'elles concernent la disparition et la réhabilitation de langues, de coutumes ou de lieux jugés «identitaires» par les «détenteurs de patrimoine» (Ciarcia 2006, Graezer Bideau 2012).

Si nous avons pu observer des visions parfois divergentes au sujet de la transmission de la langue traditionnelle et des représentations qu'elle véhicule, un consensus fort existe sur la nécessité de sauvegarde, qui touche les cercles de patoisants, les politiciens et la communauté même, et qui fonde finalement le souci patrimonial pour les langues dialectales locales, au-delà des différents niveaux d'action.

Comme on a déjà eu l'occasion de le souligner ailleurs (Diémoz, Reusser-Elzingre 2014) si ces actions patrimoniales sont en train de modifier les attitudes envers les patois, le défi sera de voir si la niche identitaire et affective qu'ils occupent actuellement pourra assurer des usages et des fonctions linguistiques complémentaires dans la communauté bi-plurilingue actuelle. Cette situation a été observée dans certaines régions linguistiques voisines (Porcellana, Diémoz 2014), ou même au niveau européen (O'Rourke et al. 2015), où des langues minoritaires qui sont encore parlées par une partie de la population ont bénéficié de politiques linguistiques mises en place par des institutions locales et par des associations qui ont réussi non seulement à améliorer le statut de ces langues mais aussi à étendre leur pratique, en particulier aux néo-locuteurs (2).

BOUDREAU A., L. DUBOIS, J. MAURAIS, G. MCCONNELL (éds.). 2002. L'écologie des langues. Paris: L'Harmattan.

GARTHOBLAZ P., G. PANNATIER (éds.). 2004. Les patois du Valais romand. Evolène: Fédération Cantonale Valaisanne des Amis du Patois.

CIARCIA G. 2006. La perte durable. Rapport d'étude sur la notion de patrimoine immatériel, Carnet du Lahic, 1.

DE PIETRO J.-F., ELMIGER D. (dir.), BERCHTOLD E., DIÉMOZ F., MAÎTRE R., REUSSER-ELZINGRE A., WÜTHRICH S. (coll.). 2012. Education et ouverture aux langues patrimoniales, Neuchâtel: IRDP.

DIÉMOZ F. 2014. Éducation et ouverture aux langues patrimoniales: de l'enseignement des parlers francoprovençaux et oiliques à la prise en compte de la variation linguistique, pp. 3-20 in Actes du 14e Colloque des langues dialectales, Monaco, 24 novembre 2012.

DIÉMOZ F., REUSSER-ELZINGRE A. 2014. Représentations linguistiques dans deux régions de Suisse romande (Jura et Valais) De la pratique du parler à la mise en patrimoine, pp. 205-224 in BONDAZ J., GRAEZER BIDEAU F., ISNART C., LEBLON A. (dir.), Local Vocabularies of «Heritage». Translations, Negotiations and Transformations. Les voca- 


\section{Les parlers francopro- vençaux de la Suisse romande: processus de patrimonialisation \\ F. DIÉMOz}

bulaires locaux du «patrimoines. Traductions, négociations et transformations, BerlinMünster-Wien-Zürich-London: Lit Verlag.

GRAEZER BIDEAU F. 2012. En Suisse comme ailleurs, il n'y a pas de traditions pures. Migros Magazine, 1, 20 (février): 24-27.

HAUGEN E. 1972. The ecology of language. Stanford CA: Stanford University Press.

HERTZ E., CHAPPAZ-WIRTHNER S. 2012. Le patrimoine a-t-il fait son temps?, wrere.Ethnographiques.org (24 juillet 2012).

KRISTOL A. 1998. Que reste-t-il des dialectes gallo-romans de Suisse romande?, pp. 101-114 in J.-M. ELOY (éd.), Evaluer la vitalité. Variétés d'oil et autres langues, Amiens: Université de Picardie-Jules Verne/Centre d'Etudes Picardes.

KRISTOL A. 1999. Histoire linguistique de la Suisse romande: quelques jalons, Babylonia 3/99: 8-13.

LÜDI G. 1990. Französisch: Diglossie und Polyglossie/Diglossie et polyglossie, pp. 307-334 in G. HOLTUS et al. (éd.), Lexikon des Romanischen Linguistik 5/1. Tübingen.

MAITRE R., MATTHEY M. 2004. Le patois d'Evolène, dernier dialecte francoprovençal parlé et transmis en Suisse, pp. 375-390 in J.-M. ELOY (éd.), Des langues collatérales. Problèmes linguistiques, sociolinguistiques et glottopolitiques de la proximité linguistique. Actes du coll. intern. réuni à Amiens, du 21 au 24 nov. 2001, volume II, Paris: L'Harmattan.

O'ROURKE B., PUJOLAR J., RAMALLO F. 2015. New speakers of minority languages: the challenging opportunity - Foreword. International fournal for the Sociology of Language No 231: 1-20.

PORCELLANA V., DIÉMOZ F. 2014. (éd.) Minoranze in mutamento. Etnicità, lingue e processi demografici nelle valli alpine italiane, Alessandria: Edizioni dell'Orso.

RAUTENBERG M. 2003. La rupture patrimoniale. Paris: A La Croisée. TELMON T., MEDELICE J. 2013. Langues et dialectes dans l'arc alpin, Education et Sociétés Plurilingues 35 (décembre): 69-79.

\section{NOTES}

(1) On désigne par néo-locuteurs ou néo-patoisants les personnes qui ont volontairement appris le patois en suivant des cours donnés par des associations ou par le biais du théâtre; les locuteurs actifs sont généralement des locuteurs dont le patois a été la première langue alors que les locuteurs passifs, tout en ayant entendu souvent le patois (par exemple en famille), le comprennent mais ne le parlent pas.

(2) Dans le cadre d'une action COST, New Speakers in a Multilingual Europe: Opportunities and Challenges (2013-2017), le contexte des langues minoritaires et de ses néo-locuteurs est un des objets d'étude au niveau européen. (http://www.cost.eu/COST_Actions/isch/IS1306, http://www.nspk.org.uk/). 